TRANSACTIONS OF THE

AMERICAN MATHEMATICAL SOCIETY

Volume 349, Number 10, October 1997, Pages 4159-4179

S 0002-9947(97)01836-9

\title{
EQUILIBRIA OF SET-VALUED MAPS ON NONCONVEX DOMAINS
}

\author{
H. BEN-EL-MECHAIEKH AND W. KRYSZEWSKI
}

\begin{abstract}
We present new theorems on the existence of equilibria (or zeros) of convex as well as nonconvex set-valued maps defined on compact neighborhood retracts of normed spaces. The maps are subject to tangency conditions expressed in terms of new concepts of normal and tangent cones to such sets. Among other things, we show that if $K$ is a compact neighborhood retract with nontrivial Euler characteristic in a Banach space $E$, and $\Phi: K \longrightarrow 2^{E}$ is an upper hemicontinuous set-valued map with nonempty closed convex values satisfying the tangency condition

$$
\Phi(x) \cap T_{K}^{r}(x) \neq \emptyset \text { for all } x \in K,
$$

then there exists $x_{0} \in K$ such that $0 \in \Phi\left(x_{0}\right)$. Here, $T_{K}^{r}(x)$ denotes a new concept of retraction tangent cone to $K$ at $x$ suited for compact neighborhood retracts. When $K$ is locally convex at $x, T_{K}^{r}(x)$ coincides with the usual tangent cone of convex analysis.

Special attention is given to neighborhood retracts having "lipschitzian behavior", called $L$-retracts below. This class of sets is very broad; it contains compact homeomorphically convex subsets of Banach spaces, epi-Lipschitz subsets of Banach spaces, as well as proximate retracts. Our results thus generalize classical theorems for convex domains, as well as recent results for nonconvex sets.
\end{abstract}

\section{INTRODUCTION}

Theorems on the existence of an equilibrium (or a zero) for a set-valued map (simply called a map) $\Phi$ from a normed space $E$ into closed subsets of $E$, required to belong to a certain constraints set $K \subset E$, are central in many nonlinear problems. Recall that an equilibrium in $K$ for the map $\Phi$ is a point $x_{0} \in K$ with $0 \in \Phi\left(x_{0}\right)$. The appellation "equilibrium" originates from the calculus of variations where $\Phi$ is typically a set of subgradients; it is also motivated by control problems where an equilibrium state $x$ is a stationary solution to a differential inclusion $x^{\prime}(t) \in \Phi(x(t))$.

The best known equilibrium result is the following theorem of B. Cornet [C], which is derived from earlier pioneering results of Ky Fan [F1], [F2] and F. Browder $[\mathrm{Br}]$.

Theorem 0. Assume that $\Phi$ is an upper semicontinuous map defined on a compact convex subset $K$ of a normed space $E$ with nonempty closed convex values in $E$. If

Received by the editors October 17, 1994 and, in revised form, March 18, 1996.

1991 Mathematics Subject Classification. Primary 47H10, 47H04; Secondary 54C55.

Key words and phrases. Equilibria, nonconvex set-valued maps, compact neighborhood retracts, normal and tangent retraction cones and inwardness, $L$-retracts.

Research supported by the Natural Sciences and Engineering Research Council of Canada under grant OGP0042422. 
$\Phi$ satisifies the tangency condition

$$
\Phi(x) \cap T_{K}(x) \neq \emptyset \text { for all } x \in K,
$$

then $\Phi$ has an equilibrium in $K$.

Here, $T_{K}(x)$ stands for the tangent cone $\overline{\bigcup_{t>0} \frac{1}{t}(K-x)}$ to $K$ at $x \in K$. Since $K$ is convex, this cone coincides with the Bouligand contingent cone, that is,

$$
T_{K}(x)=\left\{v \in E: \liminf _{t \downarrow 0^{+}} \frac{d(x+t v ; K)}{t}=0\right\},
$$

where for $z \in E, d(z ; K)=\inf _{x \in K}\|z-x\|$ is the distance from $z$ to $K$.

Since $K$ being convex is tangentially regular, $T_{K}(x)$ coincides with the Clarke tangent cone

$$
C_{K}(x):=\left\{v \in E: \limsup _{\substack{y \rightarrow x, y \in K \\ t \downarrow 0}} \frac{d(y+t v ; K)}{t}=0\right\} .
$$

The "inwardness" condition (1) may therefore be replaced by:

$$
\Phi(x) \cap C_{K}(x) \neq \emptyset \text { for all } x \in K .
$$

Theorem 0 has been refined in several directions by many authors. It was in fact formulated in $[\mathrm{C}]$ for the larger class of upper hemicontinuous maps and subsequently refined by several authors (see e.g. Lasry-Robert $[\mathrm{LR}]$ ). A coincidence point generalization was given by Ky Fan [F2]. Condition (1) was modified to include the case where $\Phi$ has values in a different normed space $F$ (see for example Aubin-Frankowska $[\mathrm{AF}]$ ) as well as in the absence of full compactness of the domain $K$ (see for instance [F2] and Lassonde [L] for various related fixed point theorems for inward set-valued maps, and Ben-El-Mechaiekh-Kryszewski [BK] for equilibrium theorems for perturbations of set-valued maps by convex processes).

It is clear that the existence of an equilibrium for the map $\Phi$ is equivalent to the existence of a fixed point for the map $i \pm \Phi$, where $i$ is the inclusion $K \hookrightarrow E$. Hence any equilibrium theorem has a fixed point counterpart and vice-versa. For instance, Theorem 0 is known to be equivalent to the Fan-Kakutani fixed point theorem (let us mention here that Theorem 0 holds true in Hausdorff locally convex topological vector spaces and even in more general spaces, e.g. spaces having sufficiently many linear functionals, or vector spaces equipped with any topology that induces the euclidean topology on finite polytopes).

Taking this observation into account and as pointed out in Clarke-Ledyaev-Stern [CLS], in generalizing criteria for the existence of equilibria for "inward" maps, one can turn to the various extensions (of homotopical or homological nature) of the Fan-Kakutani fixed point theorem to nonconvex sets. To do so, one has to clarify the important following issues:

(i) Which properties of sets are suitable substitute for convexity?

(ii) What would then be the counterpart of the tangency conditions (1) or (2)?

One of the most natural as well as convenient classes of spaces to be considered in extending results to nonconvex sets is the class of absolute retracts, and more generally that of absolute neighborhood retracts. Recall that a compact subset $K$ of a normed space $E$ is said to be a compact (neighborhood) retract of $E$ if there exists a (continuous) mapping $r: E \longrightarrow K(r: U \longrightarrow K$, where $U$ is an open neighborhood of $K$ ) such that $r(x)=x$ for all $x \in K$ (the retraction $r$ is by no means unique). 
It is well-known that every continuous self-mapping $f$ of a compact retract $K$ has a fixed point; while if $K$ is a compact neighborhood retract, then $f$ has a fixed point provided the Lefschetz number $\lambda(f)$ of $f$ is nonzero. In particular, assuming that the Euler characteristic $\chi(K) \neq 0$ (which holds true if $K$ is a retract), it follows that a map from $K$ into itself that is homotopic to the identity has a fixed point. Consequently, it is necessary to always assume that the Euler characteristic of the domain is nonzero. For, there is a tangent field with no zeros on the unit sphere $S^{1}$.

In what follows we will always assume that $K$ is a compact neighborhood retract of a Banach space $E$ (the completeness of $E$ will be needed) with a retraction $r: U \longrightarrow K$ defined on a neighborhood $U$ of $K$. Since $K$ is compact, denoting by $B(K, \delta)$ the open neighborhood of radius $\delta>0$ around $K$, we may assume with no loss of generality that:

$$
\left\{\begin{array}{c}
K \text { is a compact neighborhood retract of a Banach space } E \\
\text { with a given retraction } r: B(K, \delta) \longrightarrow K \text { for a fixed } \delta>0 .
\end{array}\right.
$$

Let us mention that the class of sets satisfying (A1) is quite large. For instance, if $K$ is compact convex then $K$ satisfies (A1) in addition to having nontrivial Euler characteristic. Furthermore, since the property of being a compact neighborhood retract and the Euler characteristic are both topological invariants, it follows that any subset $L$ of $E$ that is homeomorphic to $K$ satisfies (A1) and has nontrivial Euler characteristic, whenever $K$ has both properties. For an extensive study of retracts the reader is referred to the monographs by Borsuk $[\mathrm{Bo}]$ and $\mathrm{Hu}[\mathrm{H}]$.

We study below possible ways to address question (ii) above. The following two examples show that neither the tangency condition (1) nor (2) (which are equivalent for tangentially regular sets) are adequate for the study of equilibria in nonconvex sets.

Example 1.1. Let $K=S_{1} \cup S_{2}$, where

$$
\begin{aligned}
& S_{1}:=\left\{(x, y) \in \mathbf{R}^{2}:(x-1)^{2}+y^{2}=1\right\}, \\
& S_{2}:=\left\{(x, y) \in \mathbf{R}^{2}:(x+1)^{2}+y^{2}=1\right\} .
\end{aligned}
$$

Let $f: K \longrightarrow \mathbf{R}^{2}$ be given by the formula

$$
f(x, y):=\left\{\begin{array}{cc}
(y, 1-x), & \text { if } \quad(x, y) \in S_{1}, \\
(-y, 1+x), & \text { if } \quad(x, y) \in S_{2} .
\end{array}\right.
$$

Clearly for each $x \in K, f(x, y) \in T_{K}(x, y)=C_{K}(x, y)$; but $f$ has no zeros. It is readily seen that $K$ is compact with nontrivial Euler characteristic. Moreover $K$ is a retract of the open set $U:=\mathbf{R}^{2} \backslash\{( \pm 1,0)\}$. Indeed, write first $U=U_{1} \cup U_{2} \cup U_{3}$, where $U_{1}:=\left\{(x, y):(x-1)^{2}+y^{2} \leq 1\right.$ or $\left.(x+1)^{2}+y^{2} \leq 1\right\}$ consists of all points interior to or on $K, U_{2}:=\left\{(x, y) \notin U_{1}:|x| \leq 1\right\}$ consists of all points in the strip $|x| \leq 1$ exterior to $K$, and $U_{3}:=U \backslash\left(U_{1} \cup U_{2}\right)$. Define the continuous retraction $r: U \longrightarrow K$ radially on $U_{1} \cup U_{3}$ (points with negative abscissa are mapped radially into $S_{2}$, those with positive abscissa into $S_{1}$ ), and by vertical projection into (the nearest point of) $K$ on $U_{2}$.

Example 1.2. Let $\hat{K}=R_{1} \cup R_{2}$, where:

$$
\begin{aligned}
& R_{1}:=\left\{(x, y) \in \mathbf{R}^{2}: \frac{1}{2} \leq(x-1)^{2}+y^{2} \leq 1\right\}, \\
& R_{2}:=\left\{(x, y) \in \mathbf{R}^{2}: \frac{1}{2} \leq(x+1)^{2}+y^{2} \leq 1\right\} .
\end{aligned}
$$


Being homotopy equivalent to the set $K$ of Example 1, $\hat{K}$ is also a compact neighborhood retract with nontrivial Euler characteristic. Furthermore, $T_{\hat{K}}(x, y)=$ $C_{\hat{K}}(x, y)$ for $(x, y) \neq(0,0)$, and $T_{\hat{K}}(0,0)=\mathbf{R}^{2} \supset\{0\} \times \mathbf{R}=C_{\hat{K}}(0,0)$. One can easily extend the function $f$ of Example 1 to a function $g: \hat{K} \longrightarrow \mathbf{R}^{2}$ having no zeros.

Some remarks are now in order. Recall that (1) (stated for arbitrary sets) was used to study invariance or viability problems for differential equations (e.g. in Nagumo's theorem) or differential inclusions (see Aubin-Cellina $[\mathrm{AC}]$ and $[\mathrm{AF}]$ ). Condition (2) stated in a nonconvex context seems to be sufficient for the existence of equilibria only for restricted classes of domains, e.g. epi-Lipschitz sets or biLipschitz sets that are homeomorphic to compact convex sets (see BonnisseauCornet $[\mathrm{BC}]$ and $[\mathrm{CLS}]$ ), and more general sets considered below.

These remarks motivate us to introduce a new tangency condition suited for the study of equilibria (or fixed points) in compact neighborhood retracts. In the case where $K$ is compact and convex, our condition is slightly weaker than (1) (both conditions being equivalent when $\Phi$ has compact values). More precisely, we define a new notion of retraction normal cone $N_{K}^{r}(x)$ to a compact neighborhood retract $K$ that allows us to formulate our first main theorem (Theorem 3.5 below):

Theorem I. Assume that $K$ satisfies (A1) and has nontrivial Euler characteristic. Let $\Phi: K \longrightarrow 2^{E}$ be an upper hemicontinuous map with nonempty closed convex values such that

$$
\forall x \in K, \forall p \in N_{K}^{r}(x), \inf _{y \in \Phi(x)}\langle p, y\rangle \leq 0 .
$$

Then $\Phi$ has an equilibrium in $K$.

If $T_{K}^{r}(x):=\left[N_{K}^{r}(x)\right]^{-}$is the polar cone of $N_{K}^{r}(x)$, the condition

$$
\Phi(x) \cap T_{K}^{r}(x) \neq \emptyset \text { for all } x \in K
$$

is sufficient for (3), and equivalent to it whenever $\Phi$ has compact values.

We show that in case $K$ is locally convex at $x \in K$, then $N_{K}^{r}(x)$ coincides with the usual normal cone of convex analysis $N_{K}(x):=\left[T_{K}(x)\right]^{-}$.

We also provide convenient variations of Theorem I as well as a weaker version of that theorem for maps with nonconvex values; namely, upper semicontinuous maps with compact acyclic (with respect to Alexander-Čech cohomology with arbitrary coefficients, or Čech homology with rational coefficients) or more generally composition products of such maps.

We introduce the notion of an $\mathcal{L}$-retract: a set $K$ is said to be an $\mathcal{L}$-retract if $K$ satisfies (A1) and there exist an open neighborhood $U$ of $K$, a retraction $r: U \longrightarrow K$, and a constant $L>0$ such that

$$
\|x-r(x)\| \leq L d(x ; K) \text { for all } x \in U .
$$

We show that the property of being an $\mathcal{L}$-retract is a local property. Particular classes of domains considered in [BC], in [CLS], and proximate retracts in the sense of Plaskacz $[\mathrm{P}]$ are $\mathcal{L}$-retracts. For such sets the following holds (Theorem 5.3 below):

Theorem II. Assume that $K$ is an $\mathcal{L}$-retract with nontrivial Euler characteristic, and that $\Phi: K \longrightarrow 2^{E}$ is an upper hemicontinuous map with nonempty compact 
convex values. If condition (2) holds, that is,

$$
\Phi(x) \cap C_{K}(x) \neq \emptyset \text { for all } x \in K,
$$

then $\Phi$ has an equilibrium in $K$.

In the case when $K$ is a proximate retract (or, in the terminology of $[\mathrm{P}]$, has property $\rho$, that is, $\|x-r(x)\|=d(x ; K)$ for all $x \in U)$, we generalize to infinite dimensional spaces the equilibrium theorem of $[\mathrm{P}]$, which was, with the results in $[\mathrm{BC}]$, one of the first equilibrium theorems for a large class of nonconvex domains.

The paper is organized as follows: in Section 2 we introduce the notions of retraction normal and tangent cones and study their properties; in Section 3 we prove the first main existence result of equilibria on compact neighborhood retracts with nontrivial Euler characteristic; in Section 4 we introduce the notion of an $\mathcal{L}$-retract, study its properties and discuss various examples; in Section 5 we consider the existence of equilibria of maps defined on $\mathcal{L}$-retracts and satisfying the tangency condition (2).

Viability issues related to the existence results presented in this paper will be discussed elsewhere.

\section{Retraction Cones}

We start by fixing the notation and terminology. The closure, the interior, and the boundary of a subset $A$ of a topological space are denoted by $\bar{A}, \operatorname{int}(A)$, and $b d(A)$ respectively. By $E$ we denote a Banach space with norm $\|$.$\| . We denote by E_{s}^{*}$ and $E_{w}^{*}$ the topological dual $E^{*}$ of $E$ endowed with strong topology and the weak-* topology respectively; $\langle.,$.$\rangle is the duality pairing \langle p, x\rangle=p(x), p \in E^{*}, x \in E$. We denote by $D_{s}^{*}$ and $D_{w}^{*}$ the closed unit ball $D^{*}$ in $E^{*}$ equipped with the strong topology and the weak-* topology respectively.

Given $K \subset E, x \in K, \epsilon>0$, we put

$$
D_{K}(x, \epsilon):=\{y \in K:\|x-y\| \leq \epsilon\}, \quad B_{K}(x, \epsilon):=\{y \in K:\|x-y\|<\epsilon\} .
$$

Observe that in general the closure $\overline{B_{K}(x, \epsilon)}$ of $B_{K}(x, \epsilon)$ is contained in $D_{K}(x, \epsilon)$; equality holds e.g. when $K$ is convex.

Let $K \subset E$ be a set satisfying (A1). Then there holds:

$$
\exists \eta>0, \eta<\frac{1}{2} \delta, \forall x \in B(K, \eta),\|x-r(x)\|<\eta .
$$

Definition 2.1. For any $x \in K$ and $0<\epsilon<\eta$, let

$$
M_{K}^{r}(x ; \epsilon):=\left\{p \in D^{*}: x \in r\left[\overline{\operatorname{conv}}\left\{y \in D_{K}(x, \epsilon): \sup _{z \in \overline{B_{K}(x, \epsilon)}}\langle p, z-y\rangle \leq 0\right\}\right]\right\} .
$$

In other words, $p \in M_{K}^{r}(x ; \epsilon)$ if and only if $x$ is a fixed point for the map $K \ni x \longmapsto r\left[\overline{\operatorname{conv}}\left\{G_{\epsilon}(x, p)\right\}\right]$, where $G_{\epsilon}: K \times D^{*} \longrightarrow 2^{K}$ is the compact valued map given by

$$
G_{\epsilon}(x, p):=\left\{y \in D_{K}(x, \epsilon): \sup _{z \in \frac{B_{K}(x, \epsilon)}{B}}\langle p, z-y\rangle \leq 0\right\} .
$$

Lemma 2.1. Let $0<\epsilon<\eta$.

(i) The map $K \ni x \longmapsto D_{K}(x, \epsilon) \subset K$ is upper semicontinuous.

(ii) The map $K \ni x \longmapsto \overline{B_{K}(x, \epsilon)} \subset K$ is lower semicontinuous.

(iii) The map $G_{\epsilon}: K \times D_{w}^{*} \longrightarrow 2^{K}$ is upper semicontinuous. Hence, so is the $\operatorname{map} G_{\epsilon}: K \times D_{s}^{*} \longrightarrow 2^{K}$. 
(iv) The map $K \ni x \longmapsto M_{K}^{r}(x ; \epsilon) \subset D_{w}^{*}$ is upper semicontinuous. Hence, the map $K \ni x \longmapsto M_{K}^{r}(x ; \epsilon) \subset D_{s}^{*}$ has closed graph. In particular, the set $M_{K}^{r}(x)$ is closed in both the strong and the weak-* topologies on $D^{*}$.

Proof. (i) Clearly, if $y_{n} \rightarrow y, x_{n} \rightarrow x$, and $y_{n} \in D_{K}\left(x_{n}, \epsilon\right)$, then $y \in D_{K}(x, \epsilon)$. The upper semicontinuity follows.

(ii) The assertion follows from the fact that the map $x \longmapsto B_{K}(x, \epsilon)$ is lower semicontinuous.

(iii) It suffices to show that $G_{\epsilon}$ has closed graph. To do this, take an element $(y, x, p) \notin \operatorname{Graph}\left(G_{\epsilon}\right)$. Then either $y \notin D_{K}(x, \epsilon)$ (in which case there is an open neighborhood of $(y, x, p)$ disjoint from $\left.G r a p h\left(G_{\epsilon}\right)\right)$, or $y \in D_{K}(x, \epsilon)$ and there is $z \in \overline{B_{K}(x, \epsilon)}$ such that $\langle p, z-y\rangle=\alpha>0$. By (ii), there is a neighborhood $V$ of $x$ such that for each $x^{\prime} \in V$, there is $z^{\prime} \in \overline{B_{K}\left(x^{\prime}, \epsilon\right)}$ with $\left\|z-z^{\prime}\right\|<\frac{\alpha}{4}$. Let $S:=\left\{q \in E^{*}:|\langle q, z-y\rangle|<\frac{\alpha}{2}\right\}$. Then $T:=(p+S) \cap D^{*}$ is an open neighborhood of $p$ in $D_{w}^{*}$. Take any $x^{\prime} \in V$ and the corresponding $z^{\prime} \in \overline{B_{K}\left(x^{\prime}, \epsilon\right)}, p^{\prime} \in T$, and any $y^{\prime}$ with $\left\|y^{\prime}-y\right\|<\frac{\alpha}{4}$. Clearly,

$$
\left\langle p^{\prime}, z^{\prime}-y^{\prime}\right\rangle=\langle p, z-y\rangle+\left\langle p^{\prime}, z^{\prime}-z+y-y^{\prime}\right\rangle+\left\langle p^{\prime}-p, z-y\right\rangle>\alpha-\frac{\alpha}{2}-\frac{\alpha}{2}=0 .
$$

This shows that $(y, x, p)$ has an open neighborhood disjoint from $\operatorname{Graph}\left(G_{\epsilon}\right)$.

(iv) It suffices to observe that $p \in M_{K}^{r}(x ; \epsilon)$ if and only if $x \in r\left[\overline{\operatorname{conv}}\left\{G_{\epsilon}(x, p)\right\}\right]$. The assertion now follows from (iii).

Remark 2.2. $M_{K}^{r}(x ; \epsilon)$ is not a cone. One readily verifies that it is starshaped around zero.

We are ready now to define the retraction normal cone.

Definition 2.2. Assume that $K$ satisfies (A1). The retraction normal pre-cone at $x \in K$ is defined to be the set

$$
M_{K}^{r}(x):=\limsup _{y \rightarrow x, y \in K} M_{K}^{r}(y ; \epsilon) .
$$

More explicitly,

$$
p \in M_{K}^{r}(x) \Longleftrightarrow\left\{\begin{array}{c}
(\forall \mu>0, \forall \nu>0),\left(\exists y \in B_{K}(x, \nu), \exists \epsilon>0, \epsilon<\nu\right) \\
\text { with }\left\|p-p^{\prime}\right\|<\nu \text { for some } p^{\prime} \in M_{K}^{r}(y ; \epsilon) .
\end{array}\right.
$$

Definition 2.3. The retraction normal cone at $x \in K$ is the cone $N_{K}^{r}(x)$ spanned by $M_{K}^{r}(x)$, that is,

$$
N_{K}^{r}(x):=\left\{\lambda p \in E^{*}: p \in M_{K}^{r}(x), \lambda \in \mathbf{R}_{+}\right\} .
$$

By replacing in the above definitions the balls in $E_{s}^{*}$ by open neighborhoods of zero in $E_{w}^{*}$, we obtain the notions of weak retraction normal pre-cone and weak retraction cone at $x \in K$. More precisely, let $\mathcal{N}$ be the familty of neighborhoods of zero in $E^{*}$ equipped with the weak-* topology.

Definition 2.4. A form $p$ belongs to the weak retraction normal pre-cone denoted by $\sigma-M_{K}^{r}(x), x \in K$, if and only if:

$$
\begin{gathered}
(\forall V \in \mathcal{N}, \forall \nu>0),\left(\exists y \in B_{K}(x, \nu), \exists \epsilon>0, \epsilon<\nu\right) \\
\text { with } p \in M_{K}^{r}(y ; \epsilon)+V .
\end{gathered}
$$

The weak retraction cone denoted by $\sigma-N_{K}^{r}(x)$ is the cone spanned by $\sigma-$ $M_{K}^{r}(x)$. 
Proposition 2.3. (i) $M_{K}^{r}(x)$ and $\sigma-M_{K}^{r}(x)$ are starshaped around zero.

(ii) $N_{K}^{r}(x)$ and $\sigma-N_{K}^{r}(x)$ are closed cones in $E_{s}^{*}$ and $E_{w}^{*}$ respectively.

(iii) The maps $M_{K}^{r}, N_{K}^{r}: K \longrightarrow 2^{E_{s}^{*}}$ and $\sigma-M_{K}^{r}, \sigma-N_{K}^{r}: K \longrightarrow 2^{E_{w}^{*}}$ have closed graphs.

Proof. Only property (iii) requires a proof. It is well-known that a point $(x, y)$ belongs to the closure of the graph of a given map $F: X \longrightarrow 2^{Y}$ ( $X, Y$ being topological spaces) if $y \in \limsup _{x^{\prime} \rightarrow x} F\left(x^{\prime}\right)$. In the present context, one readily sees that $\lim \sup N_{K}^{r}\left(x^{\prime}\right) \subset N_{K}^{r}(x)$; hence if $(x, p) \in{\overline{\operatorname{Graph}\left(N_{K}^{r}\right)}}^{\left(K \times E_{s}^{*}\right)}$, then $(x, p) \in$

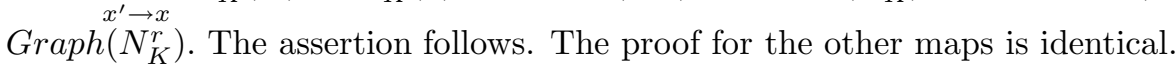

Definition 2.5. The retraction tangent cone and the weak retraction tangent cone at $x \in K$ are defined by the formulae

$$
\begin{gathered}
T_{K}^{r}(x):=\left[N_{K}^{r}(x)\right]^{-}=\left\{v \in E:\langle p, v\rangle \leq 0, \forall p \in N_{K}^{r}(x)\right\} \\
\sigma-T_{K}^{r}(x):=\left[\sigma-N_{K}^{r}(x)\right]^{-}=\left\{v \in E:\langle p, v\rangle \leq 0, \forall p \in \sigma-N_{K}^{r}(x)\right\} .
\end{gathered}
$$

As for other more usual cones, $T_{K}^{r}(x)\left(\sigma-T_{K}^{r}(x)\right)$ is a closed convex cone in $E_{s}^{*}$ ( $E_{w}^{*}$ respectively). Moreover, by Proposition 2.3 , the maps $T_{K}^{r}: K \longrightarrow 2^{E_{s}^{*}}$ and $\sigma-T_{K}^{r}: K \longrightarrow 2^{E_{w}^{*}}$ are lower semicontinuous provided the normal cones $N_{K}^{r}(x)$ and $\sigma-N_{K}^{r}(x)$ are convex for each $x \in X$ (the latter being not true in general).

Example 2.4. Assume that $x \in K$ and $K$ is locally convex at $x$, i.e. there is a neighborhood $V$ of $x$ such that $V \cap K$ is convex. Then, for sufficiently small $\epsilon>0$ $(\epsilon<\eta)$, we have

$$
M_{K}^{r}(x ; \epsilon)=D^{*} \cap N_{K}(x),
$$

where $N_{K}(x):=\left\{p \in E^{*}: \forall v \in T_{K}(x),\langle p, v\rangle \leq 0\right\}$ is the normal cone to $K$ at $x$ (in particular, if $x \in \operatorname{int}(K)$, then for small $\epsilon, M_{K}^{r}(x ; \epsilon)=\{0\}$ ).

This implies that

$$
N_{K}^{r}(x)=\sigma-N_{K}^{r}(x)=N_{K}(x) \text { and } T_{K}^{r}(x)=\sigma-T_{K}^{r}(x)=T_{K}(x) .
$$

To see this, take $\epsilon$ so that $D(x, \epsilon) \subset V$. Hence $\overline{B_{K}(x, \epsilon)}=D_{K}(x, \epsilon)$ is convex and contained in $V \cap K$. Putting $L:=D_{K}(x, \epsilon)$, we readily see that $T_{K}(x)=T_{L}(x)$, and consequently

$$
N_{K}(x)=N_{L}(x)=\left\{p \in E^{*}: \sup _{z \in L}\langle p, z\rangle=\langle p, x\rangle\right\}
$$

because $L$ is convex. Now $p \in M_{K}^{r}(x ; \epsilon)$ if and only if $x \in r\left[\overline{c o n v}\left\{G_{\epsilon}(x, p)\right\}\right]=$ $\overline{\operatorname{conv}}\left\{G_{\epsilon}(x, p)\right\}$. This holds if and only if $\langle p, x\rangle \geq \sup \{\langle p, z\rangle: z \in K\}$, that is $p \in N_{L}(x)$.

Example 2.5. Consider the set $K$ and the retraction $r$ defined in Example 1.1 of the Introduction. A quick calculation shows that $N_{K}^{r}(0,0)=\mathbf{R} \times\{0\} \cup\{0\} \times \mathbf{R}$. For the set $\hat{K}$ of Example 1.2, we have $N_{K}^{r}(0,0)=\{0\} \times \mathbf{R}$. In both situations, the dimension being finite, $N_{K}^{r}=\sigma-N_{K}^{r}$.

Retraction normal cones generally depend on the choice of the retraction, as the following example shows.

Example 2.6. Consider the compact retract of $\mathbf{R}^{3}$ defined by

$$
K:=\left\{(x, y, z) \in \mathbf{R}^{3}: x^{2}+y^{2}=z^{2}, x \leq 0,0 \leq z \leq 1\right\} .
$$


Let $K_{1}:=\left\{(x, y, z) ; x^{2}+y^{2}=1, x \leq 0\right\}$ and let $r_{1}^{\prime}: K_{1} \longrightarrow K$ be the mapping defined by

$$
r_{1}^{\prime}(x, y, z):=\text { intersection of the line }\{(x, y, \lambda z): \lambda \in \mathbf{R}\} \text { with } K .
$$

Let $K_{2}:=\{(x, y, z) ;|y| \leq z \leq 1\}$ and define a mapping $r_{2}^{\prime}: K_{2} \longrightarrow K$ by

$$
r_{2}^{\prime}(x, y, z):=\text { intersection of the line }\{(\lambda x, y, z): \lambda \in \mathbf{R}\} \text { with } K \text {. }
$$

Both mappings extend to retractions $r_{1}: \mathbf{R}^{3} \longrightarrow K, r_{2}: \mathbf{R}^{3} \longrightarrow K$. A straightforward but tedious computation shows that

$$
N_{K}^{r_{1}}(0,0,0)=\left\{(x, y, z):-z \geq|y|, x \geq \sqrt{z^{2}-y^{2}}\right\} \cup \mathbf{R}_{+} \times\{0\} \times \mathbf{R}_{+},
$$

and

$$
N_{K}^{r_{2}}(0,0,0)=\left\{(x, y, z):-z \geq|y|, x \geq \sqrt{z^{2}-y^{2}}\right\} .
$$

It is interesting to observe that the notion of normal retraction cone also depends on the choice of the norm in $E$.

Example 2.7. Let $K:=\left\{(x, y) \in \mathbf{R}^{2}: x y=0,0 \leq x, y \leq 1\right\}$ and take any retraction $r: \mathbf{R}^{2} \longrightarrow K$. Consider the norm in $\mathbf{R}^{2}$ given by $\|(x, y)\|_{a, b}:=\sqrt{\frac{x^{2}}{a^{2}}+\frac{y^{2}}{b^{2}}}, a, b>$ $0, a \neq b$. One readily shows that $N_{K}^{r}(0,0)=\mathbf{R}_{-} \times \mathbf{R}_{-} \cup \mathbf{R}_{+} \times\{0\} \cup\{0\} \times \mathbf{R}_{+} \cup\{(x, y)$ : $x, y \geq 0, a x=b y\}$ in $\left(\mathbf{R}^{2},\|\cdot\|_{a, b}\right)$.

\section{Existence of Equilibria}

The proofs of some of our main theorems rely on a generalization of the Lefschetz fixed point theorem to a large class of so-called admissible maps due to Górniewicz [G]. Roughly speaking, an upper semicontinuous map from a topological space $X$ into a topological space $Y$ is admissible if it admits a multiselection that is a finite composition of acyclic maps (recall that a map is acyclic if it upper semicontinuous and has nonempty compact acyclic values). For details, the reader is referred to $[\mathrm{G}]$, $[\mathrm{K} 1]$, [Gr] for the single-valued case, and [S] for basic notions of homology theory.

The class of admissible maps is quite large. It contains numerous subclasses of convex as well as nonconvex maps. Moreover, it satisfies basic stability properties with respect to some elementary operations. For example, we have:

Proposition 3.1 (see [G] or [K1]). (i) Any acyclic map is admissible.

(ii) The composition product of admissible maps is admissible.

(iii) Assume $Y$ is contained in a topological vector space and $\Phi, \Psi: X \longrightarrow$ $2^{Y}, \alpha: X \longrightarrow 2^{\mathbf{R}}$ are admissible maps. The maps $(\Phi+\Psi)(x):=\Phi(x)+\Psi(x)$ and $(\alpha \Phi)(x):=\alpha(x) \Phi(x), x \in X$, are admissible.

(iv) If the maps $\Phi: X \longrightarrow 2^{Y}, \Psi: X \longrightarrow 2^{Z}$ are admissible, then the map $(\Phi, \Psi)(x):=\Phi(x) \times \Psi(x) \subset Y \times Z$ is also admissible.

A Vietoris-Begle type theorem holds for admissible maps. It allows for the definition of a generalized Lefschetz "set" $\Lambda$ (.) for such maps; which for acyclic maps reduces to a singleton, and coincides with the classical Lefschetz number $\lambda($.$) for single-valued mappings. Naturally, this generalized Lefschetz number is a$ homotopy invariant. More precisely, given two admissible maps $\Phi, \Psi: X \longrightarrow 2^{Y}$, one has $\Lambda(\Phi) \cap \Lambda(\Psi) \neq \emptyset$ whenever $\Phi$ and $\Psi$ are homotopic through an admissible homotopy $\Xi: X \times[0,1] \longrightarrow 2^{Y}$ with $\Xi(., 0)=\Phi($.$) and \Xi(., 1)=\Psi($.$) .$ 
A Lefschetz-type theorem holds for admissible maps: if $\Phi$ is an admissible map of a compact absolute neighborhood retract $K$ such that $\Lambda(\Phi) \neq\{0\}$, then $\Phi$ has a fixed point.

Assume now that $K$ satisfies (A1) and that $H$ is the Alexander-Čech cohomology theory with rational coefficients. Then, the graded linear space $H(K)$ is of finite type, i.e. $H^{q}(K)=0$ for almost all $q \geq 0$ and $\operatorname{dim}_{\mathbf{Q}} H^{q}(K)<\infty$ for all $q \geq 0$. The Euler characteristic of $K$ is defined as $\chi(K):=\Lambda(i d)=\lambda(i d)=\sum(-1)^{q} \beta_{q}(K)$, where $\beta_{q}(K):=\operatorname{dim}_{\mathbf{Q}} H^{q}(K)$ is the $q$-th Betti number of $K$; here $i d$ is the identity mapping on $K$. The following result is an immediate consequence of the Lefschetz property we have just formulated.

Theorem 3.2 (see $[\mathrm{G}]$ or $[\mathrm{K} 1]$ ). Assume that $K$ is a compact absolute neighborhood retract with $\chi(K) \neq 0$. If $\Phi: K \longrightarrow 2^{K}$ is an admissible map homotopic to the identity, then $\Phi$ has a fixed point.

Example 3.3. Assume that $K \subset E$ satisfies (A1). If $\Phi: K \longrightarrow 2^{K}$ is an admissible map with a selected pair (see see $[\mathrm{G}]$ or $[\mathrm{K} 1])(u, v)$ such that

$$
\|u(y)-v(y)\|<\delta \quad \text { for each } y \in \Gamma,
$$

then $\Phi$ has a fixed point provided $\chi(K) \neq 0$.

To see this, consider the admissible map $\Xi: K \times[0,1] \longrightarrow 2^{K}$ defined by the formula

$$
\Xi(x, t):=V\left(U^{-1}(x, t)\right),
$$

where $U, V: \Gamma \times[0,1] \longrightarrow K$ are given by

$$
U(y, t):=(u(y), t) \text { and } V(y, t):=r((1-t) v(y)+t u(y)), \quad y \in \Gamma, t \in[0,1] .
$$

The map $U$ is a Vietoris map, and the map $V$ is well-defined since

$$
d((1-t) v(y)+t u(y) ; K) \leq\|(1-t) v(y)+t u(y)-v(y)\|=t\|u(y)-v(y)\|<\delta,
$$

for any $y \in \Gamma$ and $t \in[0,1]$. Therefore $(U, V)$ is a selected pair for $\Xi$. One readily verifies that $\Xi(x, 0)=\Phi(x)$ and $\Xi(x, 1)=\Psi(x)$ for $x \in K$. Hence, $\Lambda(\Phi) \ni \lambda(i d)=$ $\chi(K) \neq 0$. The assertion follows from Theorem 3.2.

Example 3.4. Assume that $\Phi: K \longrightarrow 2^{K}$ is an admissible map such that, for every $x \in K, \Phi(x) \subset D(x, \epsilon)$ for some $\epsilon \in(0, \delta)$. Then condition (5) of the preceding example is satisfied.

Indeed, take any selected pair $(u, v)$ of $\Phi$, any $y \in \Gamma$, and let $x=u(y)$, i.e. $y \in u^{-1}(x)$. Hence, $\|u(y)-v(y)\|=\|x-v(y)\| \leq \epsilon<\delta$.

Recall that a map $\Phi$ from a topological space $X$ into a topological vector space $Y$ is said to be upper hemicontinuous if for every bounded linear form $p$ on $Y$, the extended real function $x \longmapsto \sup \{\langle p, y\rangle: y \in \Phi(x)\}$ is upper semicontinuous. Any upper semicontinuous map from $X$ into $Y$ supplied with the weak topology is upper hemicontinuous. Both continuity concepts coincide for maps with convex weakly compact values.

We are ready to state and prove the main theorem of this section.

Theorem 3.5. Assume that $K$ satisfies (A1) and $\chi(K) \neq 0$. If $\Phi: K \longrightarrow 2^{E}$ is upper hemicontinuous with closed convex values and satisfies (3), i.e.

$$
\forall x \in K, \forall p \in N_{K}^{r}(x), \inf _{y \in \Phi(x)}\langle p, y\rangle \leq 0,
$$

then $\Phi$ has an equilibrium. 
Proof. Suppose that $0 \notin \Phi(x)$ for each $x \in K$. By the separation theorem, for each $x \in K$ there is $p_{x} \in E^{*}$ such that $\inf \left\{\left\langle p_{x}, y\right\rangle: y \in \Phi(x)\right\}>0$, i.e. $\sup _{y \in \Phi(x)}\left\langle-p_{x}, y\right\rangle$ $<0$. Since $\Phi$ is upper hemicontinuous, the set

$$
U(x):=\left\{z \in K: \sup _{y \in \Phi(z)}\left\langle-p_{x}, y\right\rangle<0\right\}
$$

is an open neighborhood of $x$, and the collection $\mathcal{U}:=\{U(x)\}_{x \in K}$ constitutes an open covering of $K$. Let $\left\{\lambda_{x}\right\}_{x \in K}$ be a locally finite partition of unity subordinated to $\mathcal{U}$. Let us define a continuous map $f: K \longrightarrow E_{s}^{*}$ by the formula

$$
f(z):=\sum_{x \in K} \lambda_{x}(z) p_{x}, \text { for } z \in K .
$$

Then, for any $z \in K$, we have $\sup _{y \in \Phi(z)}\langle-f(z), y\rangle<0$; hence, $f(z) \neq 0$. Indeed, let $\left\{x_{1}, \ldots, x_{k}\right\}=\{x \in K: z \in U(x)\}$. Then $f(z)=\sum_{i=1}^{k} \lambda_{i}(z) p_{i}$ where $\lambda_{i}=\lambda_{x_{i}}, p_{i}=$ $p_{x_{i}}, i=1, \ldots, k$. Since $z \in U\left(x_{i}\right)$, it follows that $\sup _{y \in \Phi(z)}\left\langle-p_{i}, y\right\rangle<0$, and we are done.

We are going to prove the existence of an element $\bar{x} \in K$ such that $f(\bar{x}) \in N_{K}^{r}(\bar{x})$. This together with conditions (3) and (5) will lead to a contradiction.

Let $n \in \mathbf{N}$ be such that $0<\frac{1}{n}<\eta$, where $\eta$ is given by (A2). Consider the map $\Psi_{n}: K \longrightarrow 2^{K}$ defined by

$$
\Psi_{n}(x):=r\left[\overline{\operatorname{conv}}\left\{G_{\frac{1}{n}}\left(x, \frac{f(x)}{\|f(x)\|}\right)\right\}\right], \text { for } x \in K
$$

(see Definition 2.1). According to Lemma 2.1 (iii) and Proposition 3.1 (ii)-(iii), the map $\Psi_{n}$ is admissible. Moreover, for any $x \in K, \Psi_{n}(x) \in D(x, 2 \eta)$. Indeed, if $y \in$ $\Psi_{n}(x)$ then $y=r(z), z \in \operatorname{conv}\left\{G_{\frac{1}{n}}(x, f(x))\right\} \subset D\left(x, \frac{1}{n}\right) \subset D(x, \eta)$. Therefore, in view of (A2), $\|y-x\| \leq\|r(z)-z\|+\|z-x\| \leq 2 \eta<\delta$. Since $\Psi_{n}$ satisfies all conditions of Example 3.4, we infer that there is $x_{n} \in \Psi_{n}\left(x_{n}\right)$. In other words $\frac{f\left(x_{n}\right)}{\left\|f\left(x_{n}\right)\right\|} \in$ $M_{K}^{r}\left(x_{n} ; \frac{1}{n}\right)$. The compactness of $K$ implies that (without loss of generality) $x_{n} \rightarrow \bar{x} \in$ $K$ as $n \rightarrow \infty$. Hence $f\left(x_{n}\right) \rightarrow f(\bar{x}) \in N_{K}^{r}(\bar{x})$.

Since (4) implies (3), we have

Corollary 3.6. Suppose that $K$ and $\Phi$ are as in Theorem 3.5 and

$$
\Phi(x) \cap T_{K}^{r}(x) \neq \emptyset, \text { for all } x \in K .
$$

Then $\Phi$ has an equilibrium.

Remark 3.7. Taking into account Example 2.4, we see that in case $K$ is compact convex, Corollary 3.6 is equivalent to Theorem 0 of the Introduction.

Let us observe that condition (3) (or (4)) prescribes a direction for $\Phi(x)$ for each $x \in K$. However, for some $x \in K$, the cone $N_{K}^{r}(x)$ may be quite wide (or equivalently, $T_{K}^{r}(x)$ may be nearly $\left.\{0\}\right)$, in which case the tangency condition seems restrictive. This motivates us to consider a condition (that seems new even in the convex setting) which, unlike tangency or normality conditions, forbids certain directions to the map $\Phi$. Though this condition seems most appealing for singlevalued maps on finite dimensional spaces, it applies also to set-valued maps. 
Theorem 3.8. Assume that $K \subset E$ satisfies (A1), and let $\Phi: K \longrightarrow 2^{E}$ be an admissible map such that

$$
\forall x \in K, \forall p \in \sigma-N_{K}^{r}(x), \forall y \in \Phi(x),(p, y \neq 0 \Rightarrow\langle p, y\rangle<\|p\|\|y\|) .
$$

Then $\Phi$ has an equilibrium provided $\chi(K) \neq 0$.

Before giving the proof, let us assume that $E$ is a Hilbert space (e.g. $E=\mathbf{R}^{n}$ ). Condition (6) asserts that for any given $x \in K, p \in \sigma-N_{K}^{r}(x),\left\{\lambda p: \lambda \in \mathbf{R}_{+}\right\} \cap$ $\Phi(x) \subseteq\{0\}$. In other words, no nontrivial direction generated by $\Phi(x)$ belongs to $\sigma-N_{K}^{r}(x)\left(N_{K}^{r}(x)\right.$ in case $\left.E=\mathbf{R}^{n}\right)$.

Proof. Assume that $0 \notin \Phi(x)$ for every $x \in K$. Consider the duality map $J$ : $E \backslash\{0\} \longrightarrow 2^{D^{*}}$ defined by

$$
J(z):=\left\{p \in D^{*}:\langle p, z\rangle=\|z\|\right\} .
$$

We shall show that $J: E \longrightarrow 2^{D_{w}^{*}}$ is an acyclic map. To do this, it suffices to show that $\operatorname{Graph}(J)$ is closed, because if $p \in J(z)$, then $\|p\|=1$. Hence since $D_{w}^{*}$ is compact, the values of $J$ are compact; being in addition convex, they are acyclic.

Let $(z, p) \notin \operatorname{Graph}(J)$, i.e. $z \in E, p \in D^{*}$, and $\langle p, z\rangle=\alpha<\|z\|$. Let $\beta:=\|z\|-\alpha$ and $V:=\left\{q \in E^{*}:|\langle q, z\rangle|<\frac{\beta}{4}\right\}$. Thus $T:=(p+V) \cap D^{*}$ is an open neighborhood of $p$ in $D_{w}^{*}$. Take $p^{\prime} \in T$ and $z^{\prime}$ with $\left\|z-z^{\prime}\right\|<\frac{\beta}{4}$. It follows that

$$
\left\langle p^{\prime}, z^{\prime}\right\rangle=\langle p, z\rangle+\left\langle p^{\prime}-p, z\right\rangle+\left\langle p^{\prime}, z^{\prime}-z\right\rangle<\alpha+\frac{\beta}{2}=\|z\|-\frac{\beta}{2} \leq\left\|z^{\prime}\right\|,
$$

i.e. $\left(z^{\prime}, p^{\prime}\right) \notin \operatorname{Graph}(J)$. Therefore $J$ is upper semicontinuous.

By Proposition 3.1 (i)-(ii), the composite map $\Psi=J \circ \Phi: K \longrightarrow 2^{D_{w}^{*}}$ is admissible. Take an arbitrary $\epsilon>0, \epsilon<\eta$, where $\eta$ is provided by (A2), and consider the map $G_{\epsilon}: K \times D_{w}^{*} \longrightarrow 2^{K}$ defined in Section 2. According to Lemma 2.1 (iii), $G_{\epsilon}$ is u.s.c. Hence, by Proposition 3.1 (ii)-(iii), the map $\Psi_{\epsilon}: K \longrightarrow 2^{K}$ given by

$$
\Psi_{\epsilon}(x):=r\left[\overline{\operatorname{conv}}\left\{G_{\epsilon}(\{x\} \times \Psi(x))\right\}\right] \text {, for all } x \in K,
$$

is admissible. Arguing as in the proof of Theorem 3.5, we obtain that $\Psi_{\epsilon}$ has a fixed point $x_{\epsilon}$, that is, $x_{\epsilon} \in r\left[\overline{c o n v}\left\{G_{\epsilon}\left(x, p_{\epsilon}\right)\right\}\right]$ for some $p_{\epsilon} \in \Psi\left(x_{\epsilon}\right)$, i.e. $p_{\epsilon} \in M_{K}^{r}\left(x_{\epsilon}\right)$. The compactness of $K \times D_{w}^{*}$ implies that a subnet of $\left(x_{\epsilon}, p_{\epsilon}\right)_{0<\epsilon<\eta}$ converges to some $(\bar{x}, \bar{p}) \in K \times D^{*}$. The upper semicontinuity of $\Psi$ implies that $\bar{p} \in \Psi(\bar{x})$. Consequently, $\|\bar{p}\|=1$, and there exists $\bar{z} \in \Phi(\bar{x})$ with $\langle\bar{p}, \bar{z}\rangle=\|\bar{z}\|$. On the other hand, since $p_{\epsilon} \in M_{K}^{r}\left(x_{\epsilon}\right)$, it follows that $\bar{p} \in \sigma-M_{K}^{r}(\bar{x}) \subset \sigma-N_{K}^{r}(\bar{x})$. This is a contradiction in view of (6).

In a finite dimensional setting, this theorem has the following alternative form.

Corollary 3.9. Assume that $K \subset \mathbf{R}^{n}$ is a compact neighborhood retract with $\chi(K) \neq 0$. Given an arbitrary neighborhood retraction $r$ and an admissible map $\Phi: K \longrightarrow 2^{\mathbf{R}^{n}}$, one of the following conditions is satisfied:

(i) $\Phi$ has no zeros in $K$;

(ii) there exists $x \in K$ with $\Phi(x) \cap N_{K}^{r}(x) \neq \emptyset$.

We leave it to the reader to state equivalent results on the existence of fixed points subject to inwardness (or outwardness) conditions in the spirit of Ky Fan [F2]. We state only the following interesting result. 
Theorem 3.10. Assume that $K \subset \mathbf{R}^{n}$ is a compact neighborhood retract with $\chi(K) \neq 0$, and let $\Phi: K \longrightarrow 2^{\mathbf{R}^{n}}$ be an admissible map such that $\Phi(x)$ has no common points with $x-N_{K}^{r}(x)$ except possibly 0 (here $r$ is an arbitrary neighborhood retraction on $K)$. Then $\Phi$ has a fixed point.

\section{4. $\mathcal{L}$-RETRACTS}

Let $(X, d)$ be a fixed metric space.

We say that a subset $K$ of $X$ is an $\mathcal{L}$-retract (of $X$ ) if there are a neighborhood $U$ of $K$ in $X$, a retraction $r: U \longrightarrow K$, and a constant $L>0$ such that

$$
d(r(x), x) \leq L d(x ; K) \text { for all } x \in U
$$

Clearly, any $\mathcal{L}$-retract is a neighborhood retract of $X$ and, in particular, is closed in $X$.

In Section 5 below, we shall study equilibria of maps defined on an $\mathcal{L}$-retract $K$ of a normed space $E$. But first, let us show that the class of $\mathcal{L}$-retracts is quite important and contains examples of nonconvex sets studied elsewhere in the context of the theory of equilibria.

Example 4.1. Suppose that $A$ is a closed subset of $X$ that is bi-Lipschitz homeomorphic to a closed convex subset $B$ of a normed space $(F,\|\|$.$) (cf. [CLS]),$ i.e. there exists a Lipschitz homeomorphism $h: A \longrightarrow B$ with Lipschitz inverse $g=h^{-1}$. We claim that $A$ is an $\mathcal{L}$-retract.

To see this, consider the so-called Dugundji system $\left\{U_{s}, a_{s}\right)_{s \in S}$ for $X \backslash A$ (see $[\mathrm{D}]$ or $[\mathrm{Bo}])$, i.e.:

(i) $U_{s} \subset X \backslash A, a_{s} \in A, s \in S$;

(ii) $\left\{U_{s}\right\}_{s \in S}$ is a locally finite open cover of $X \backslash A$;

(iii) if $x \in U_{s}$, then $d\left(x, a_{s}\right) \leq 2 d(x ; A), s \in S$.

Such systems always exist (see e.g. [Bo]). Let $f: X \longrightarrow B$ be the continuous extension of the map $h$ to the entire space $X$, defined in $[\mathrm{A}]$ by the formula

$$
f(x):= \begin{cases}h(x) & \text { for } x \in A \\ \sum_{s \in S} \lambda_{s}(x) h\left(a_{s}\right) & \text { for } x \in X \backslash A,\end{cases}
$$

where $\left\{\lambda_{s}\right\}_{s \in S}$ is a locally finite partition of unity subordinated to $\left\{U_{s}\right\}_{s \in S}$.

Let $r: X \longrightarrow A$ be given by

$$
r(x):=(g \circ f)(x), \quad x \in X .
$$

We show that (A3) is satisfied; more precisely, $d(r(x), x) \leq L d(x ; A)$ for each $x \in X$, where $L=3 L_{g} L_{h}+1, L_{g}$ and $L_{h}$ being the Lipschitz constants of $g$ and $h$ respectively.

Indeed, for any given $x \in X$, if $S(x)=\left\{s \in S: x \in U_{s}\right\}$ then $f(x)=$ $\sum_{s \in S(x)} \lambda_{s}(x) h\left(a_{s}\right)$. Take $s \in S(x), \epsilon>0$ arbitrary, and $a \in K$ such that

$$
d(x ; A) \leq d(x, a)<d(x ; A)+\epsilon .
$$

Since $d\left(a_{s}, a\right) \leq d\left(a_{s}, x\right)+d(x, a) \leq 3 d(x ; A)+\epsilon$, it follows that

$$
\|f(x)-f(a)\| \leq \sum \lambda_{s}(x)\left\|h\left(a_{s}\right)-h(a)\right\| \leq L_{h}(3 d(x ; A)+\epsilon)
$$

and

$$
\begin{aligned}
d(r(x), x) & \leq d((g \circ f)(x),(g \circ f)(a))+d(a, x) \\
& \leq L_{g} L_{h}(3 d(x ; A)+\epsilon)+d(x ; A)+\epsilon .
\end{aligned}
$$


Remark 4.2. In the particular case where $A=B, h=g=i d$, and $L_{g}=L_{h}=1$, for any given number $\lambda \in(1,2)$, one can construct a retraction $r: F \longrightarrow A$ such that $\|r(x)-x\| \leq \lambda d(x ; A)$. If $F$ is a Hilbert space, $\lambda$ can be taken to be equal to 1 .

Example 4.3. In $[\mathrm{P}]$ the author considers the class of subsets $K \subset \mathbf{R}^{n}$ having property $\rho$, i.e. such that there are a neighborhood $U$ of $K$ and a retraction $r: U \longrightarrow K$ with $\|r(x)-x\|=d(x ; K)$. It is shown there that, for example, any $C^{2}$-submanifold of $\mathbf{R}^{n}$ has property $\rho$. Obviously, each set $K \subset \mathbf{R}^{n}$ having property $\rho$ is an $\mathcal{L}$-retract.

Example 4.4. Suppose that $K \subset X$ is a neighborhood retract with Lipschitz continuous retraction $r: U \longrightarrow K$. Then,

$$
\text { for each } x \in U, d(r(x), x) \leq(L+1) d(x ; K),
$$

where $L>0$ is the Lipschitz constant of $r$.

Indeed, take any $\epsilon>0$ and $y \in K$ such that $d(x, y)<d(x ; K)+\epsilon$. Therefore

$$
\begin{gathered}
d(r(x), x) \leq d(r(x), y)+d(x, y)=d(r(x), r(y))+d(x, y) \\
\leq(L+1) d(x, y)<(L+1)(d(x ; K)+\epsilon) .
\end{gathered}
$$

Remark 4.5. Observe that in case $K$ is compact, for $K$ to be an $\mathcal{L}$-retract, it suffices that the retraction $r$ be locally Lipschitz continuous.

To see this, assume that $V$ is an open neighborhood of $K$ on which a retraction $r$ is locally Lipschitz, i.e. for each $x \in V$ there is $\epsilon(x)>0$ such that the restriction of $r$ to the open ball $B(x, 2 \epsilon(x))$ is Lipschitz continuous with constant $L(x)>0$. By compactness, there are $x_{1}, \ldots, x_{k} \in K$ such that $K \subset \bigcup_{i=1}^{k} B\left(x_{i}, \epsilon\left(x_{i}\right)\right)$. Let $L=\max _{1 \leq i \leq k} L\left(x_{i}\right)$ and let $0<\epsilon \leq \min _{1 \leq i \leq k} \epsilon\left(x_{i}\right)$ be such that $U=B(K, \epsilon) \subset V$. For any $x \in U$ take $y \in K$ and $x_{i}, i=1, \ldots, k$, such that $d(x, y)=d(x ; K)$ and $y \in B\left(x_{i}, \epsilon\left(x_{i}\right)\right)$. Thus $x, y \in B\left(x_{i}, 2 \epsilon\left(x_{i}\right)\right)$ and $d(r(x), r(y)) \leq L\left(x_{i}\right) d(x, y)$; hence $d(r(x), x) \leq\left(L\left(x_{i}\right)+1\right) d(x, y) \leq(L+1) d(x ; K)$.

This last remark leads to the next new notion and proposition, which constitute a source of examples of $\mathcal{L}$-retracts.

Definition 4.1. We say that a metric space $Y$ has the neighborhood Lipschitz extension property with respect to $X(N L E P(X))$ if, for any closed subset $A \subset X$ and any locally Lipschitz continuous map $f: A \longrightarrow Y$, there is a locally Lipschitz continuous extension of $f$ on some open neighborhood of $A$.

Before studying this notion, let us make the following

Remark 4.6. It is obvious that $N L E P(X)$ is invariant under bi-Lipschitz homeomorphisms. More precisely, let $Y, Y^{\prime}$ be bi-Lipschitz homeomorphic to each other. If $Y$ has $N L E P(X)$, so does $Y^{\prime}$.

Proposition 4.7. (i) Assume that $K \subset X$ is compact and has $N L E P(X)$. Then $K$ is an $\mathcal{L}$-retract.

(ii) Let $K$ be a closed subset of a normed space. If there exists a locally Lipschitz neighborhood retraction $r: U \longrightarrow K(U$ is an open neighborhood of $K)$, then $K$ has $N L E P(X)$.

Proof. (i) Any locally Lipschitz continuous neighborhood extension of the identity on $K$ is the desired locally Lipschitz retraction. Example 4.4 and Remark 4.5 end the proof. 
(ii) Let $A$ be closed in $X$ and $f: A \longrightarrow K$ be locally Lipschitz. As in Example 4.1, we construct an extension $f^{*}: X \longrightarrow F$ of $f$ by the formula

$$
f^{*}(x):=\sum_{s \in S} \lambda_{s}(x) f\left(a_{s}\right), \quad x \in X,
$$

where $\left\{\lambda_{s}\right\}_{s \in S}$ is a locally Lipschitz continuous partition of unity. Thus, any $x \in$ $(X \backslash A) \cup \operatorname{int}(A)$ has a neighborhood on which $f^{*}$ is Lipschitz continuous.

Given $a_{0} \in b d(A)$, there is $\delta_{0}>0$ such that $f$ is Lipschitz (with Lipschitz constant $\left.L_{0}\right)$ on $V_{0}=B\left(a_{0}, \delta_{0}\right) \cap A$. Let $a, x \in B\left(a_{0}, \frac{\delta_{0}}{3}\right)$ and $a \in A$. If $x \in U_{s}$, then

$$
d\left(a_{0}, a_{s}\right) \leq d\left(a_{s}, x\right)+d\left(x, a_{0}\right) \leq 2 d(x ; A)+d\left(x, a_{0}\right) \leq 3 d\left(x, a_{0}\right)<\delta_{0},
$$

and hence $a, a_{s} \in V_{0}$. It follows that

$$
\begin{aligned}
\left\|f^{*}(x)-f^{*}(a)\right\| & =\left\|f^{*}(x)-f(a)\right\| \leq \sum \lambda_{s}(x)\left\|f\left(a_{s}\right)-f(a)\right\| \\
& \leq L_{0} d\left(a_{s}, a\right) \leq 3 L_{0} d(x, a) .
\end{aligned}
$$

Altogether, $f^{*}$ is locally Lipschitz on $X$. Let $V=f^{*-1}(U)$; the map $\bar{f}=r \circ\left(f^{*} \mid V\right)$ is the required locally Lipschitz neighborhood extension of $f$.

Remark 4.8. In Proposition 4.7 (ii), one only needs $K$ to be a locally Lipschitz neighborhood retract of some closed convex subset $C$ of a normed space.

Example 4.9. Suppose that $C$ is a closed (or open) convex subset of a normed space $F$, and $g: C \longrightarrow \mathbf{R}$ is a Lipschitz function with epigraph $\operatorname{Epi}(g)$. Let $z=$ $(x, g(x)) \in \operatorname{Graph}(g), x \in C$. Any open neighborhood $V$ of $z$ in $C \times \mathbf{R}$ contains a closed (in $F \times \mathbf{R}$ ) neighborhood $U$ of $z$ such that $U \cap \operatorname{Epi}(g)$ has $N L E P(X)$.

Indeed, since $V$ is open in $C \times \mathbf{R}$, we can find numbers $\epsilon, \delta>0$ such that $g\left(D_{C}(x, \epsilon)\right) \subset J:=[g(x)-\delta, g(x)+\delta]$ (where, as above, $D_{C}(x, \epsilon):=\{y \in C:$ $\|x-y\| \leq \epsilon\})$ and such that $D(x, \epsilon):=\{y \in F:\|x-y\| \leq \epsilon\} \subseteq C$ in case $C$ is open (in which case $D_{C}(x, \epsilon)=D(x, \epsilon)$ ). The set $U:=D_{C}(x, \epsilon) \times J$ is a closed convex neighborhood of $z$, and there is a Lipschitz retraction $r: U \longrightarrow U \cap \operatorname{Epi}(g)$, namely

$$
r(x, \lambda):=\left\{\begin{array}{ll}
(x, \lambda) & \text { when } g(y) \leq \lambda, \\
(y, g(y)) & \text { otherwise },
\end{array} \text { for all }(x, \lambda) \in U .\right.
$$

Clearly, $r$ is a retraction; since $g$ is Lipschitz, so is $r$. Proposition 4.7 (ii) and Remark 4.8 end the proof.

Example 4.10. One readily verifies that for any closed disk $D$ in a normed space $F$ there is a Lipschitz retraction $r: F \longrightarrow D$. Proposition 4.7 hence implies that $D$ has $N L E P(X)$.

Lemma 4.11. (i) If a metric space $Y$ has $N L E P(X)$ and $Y_{0}$ is open in $Y$, then $Y_{0}$ has $N L E P(X)$.

(ii) Assume that $Y:=Y_{1} \cup Y_{2}$ where $Y_{i}$ is open in $Y$ and $Y_{i}$ has $N \operatorname{LEP}(X)$, $i=1,2$. Then $Y$ has $N L E P(X)$.

Proof. (i) Let $A$ be closed in $X$ and $f: A \longrightarrow Y_{0}$ be locally Lipschitz continuous. There are an open neighborhood $U^{\prime}$ of $A$ and a locally Lipschitz continuous map $f^{*}: U^{\prime} \longrightarrow Y$ such that $f^{*} \mid A=f$. Let $U:=f^{*-1}\left(Y_{0}\right)$. The restriction $\bar{f}=f^{*} \mid U$ is the required locally Lipschitz extension of $f$.

(ii) Let $A$ be closed in $X$ and $f: A \longrightarrow Y$ be locally Lipschitz continuous. Assume first that $Y_{1} \cap Y_{2}=\emptyset$. Then $A=A_{1} \cup A_{2}$, where $A_{i}$ is closed and contained in $f^{-1}\left(Y_{i}\right), i=1,2$. Since $A_{1} \cap A_{2}=\emptyset$, for $i=1,2$, there are two disjoint open 
neighborhoods $U_{i}$ of $A_{i}$, and locally Lipschitz maps $f_{i}: U_{i} \longrightarrow Y_{i}$ such that $f_{i} \mid A_{i}=$ $f \mid A_{i}$. Let $U=U_{1} \cup U_{2}$ and define $\bar{f}: U \longrightarrow Y$ by the formula $\bar{f}(x):=f_{i}(x)$ for $x \in U_{i}, i=1,2$. The map $\bar{f}$ is the required Lipschitz neighborhood extension of $f$.

Now let $Y_{0}=Y_{1} \cap Y_{2} \neq \emptyset$, and let $\left\{A_{1}, A_{2}\right\}$ be a closed cover of $A$ such that $A_{i} \subset f^{-1}\left(Y_{i}\right), i=1,2$. Let $H$ be an open subset of $A$ such that

$$
A_{0}=A_{1} \cap A_{2} \subset H \subset \bar{H} \subset f^{-1}\left(Y_{0}\right) .
$$

By (i), $Y_{0}$ has $N L E P(X)$. Hence, there exist an open subset $U$ of $X$ and a locally Lipschitz map $f_{0}^{*}: U \longrightarrow Y_{0}$ such that $\bar{H} \subset U$ and $f_{0}^{*}|\bar{H}=f| \bar{H}$. Choose open subsets $W$ and $W^{\prime}$ of $X$ such that $W \subset \bar{W} \subset W^{\prime} \subset \bar{W}^{\prime} \subset U$ and

$$
A_{0} \subset W \cap A \subset \bar{W} \cap A \subset W^{\prime} \cap A \subset \bar{W}^{\prime} \cap f^{-1}\left(Y_{0}\right)=\bar{W}^{\prime} \cap A \subset \bar{H} .
$$

Clearly, the sets $A_{i} \backslash W, i=1,2$, are closed and disjoint. Hence, there are disjoint open subsets $V_{1}, V_{2}$ of $X$ such that:

$$
A_{i} \backslash W \subset V_{i} \text { and } A_{i} \cap V_{j}=\emptyset \text { for } i, j=1,2, i \neq j .
$$

Now define $\bar{f}_{i}: \bar{W}^{\prime} \cup A_{i} \longrightarrow Y_{i}$ by

$$
\bar{f}_{i}(x):= \begin{cases}f_{0}^{*}(x) & \text { for } x \in \bar{W}^{\prime} \\ f(x) & \text { for } x \in A_{i}, \quad i=1,2 .\end{cases}
$$

Since $\bar{W}^{\prime} \cap A_{i} \subset \bar{H}$, if $x \in \bar{W}^{\prime} \cap A_{i}$, then $f_{0}^{*}(x)=f(x)$. This implies the continuity of $\bar{f}_{i}$. Since $\bar{W} \subset W^{\prime}$, it follows that the maps $f_{i}=\bar{f}_{i} \mid W \cup A_{i}, i=1,2$, are locally Lipschitz. Since $Y_{i}$ have the $N L E P(X)$, there are open subsets $W_{i}$ of $X$ and locally Lipschitz maps $f_{i}^{*}: W_{i} \longrightarrow Y_{i}$ such that $\bar{W} \cup A_{i} \subset W_{i}$ and $f_{i}^{*}$ extends $f_{i}, i=1,2$.

Let us put

$$
U_{0}=W \cup\left(W_{1} \cap V_{1}\right) \cup\left(W_{2} \cap V_{2}\right),
$$

and define $\bar{f}: U_{0} \longrightarrow Y$ by

$$
\bar{f}(x):= \begin{cases}f_{i}^{*}(x) & \text { for } x \in W_{i} \cap V_{i}, \quad i=1,2 . \\ f_{0}^{*}(x) & \text { for } x \in W,\end{cases}
$$

Clearly, $U_{0}$ is an open neighborhood of $A$ and $\bar{f} \mid A=f$. Since $V_{1} \cap V_{2}=\emptyset$, if $x \in W \cap\left[\left(W_{1} \cap V_{1}\right) \cup\left(W_{2} \cap V_{2}\right)\right]$ then $x$ belongs to exactly one set $W \cap W_{i} \cap V_{i}, i \in$ $\{1,2\}$, and $f_{i}^{*}(x)=f_{0}^{*}(x)$. This implies the continuity of $\bar{f}$. Clearly, $\bar{f}$ is also locally Lipschitz continuous.

Theorem 4.12. If $Y$ is a compact metric space in which every point has a neighborhood that has $N \operatorname{LEP}(X)$, then $Y$ has $N L E P(X)$.

Proof. The hypothesis combined with Lemma 4.11 (i) yields the existence of a finite cover $\left\{Y_{i}\right\}_{i=1}^{k}$ of $Y$ consisting of open sets $Y_{i}, i=1, \ldots, k$, that have the $N L E P(X)$. Repeated use of Lemma 4.11 (ii) implies that $Y$ has $N L E P(X)$.

Generalizing definitions in $[R]$ and [CLS], we say that

Definition 4.2. A subset $B$ of a normed space $F$ is an epi-Lipschitz set if each point $y \in b d(B)$ has a neighborhood $U$ (in $B$ ) for which there exist a normed space $F^{\prime}$, an open set $C \subset F^{\prime}$, a Lipschitz function $g: C \longrightarrow \mathbf{R}$, a point $z=(x, g(x)), x \in$ $C$, a neighborhood $V$ of $z$, and a bi-Lipschitz homeomorphism $h: V \cap E p i(g) \longrightarrow U$ such that $h(z)=y$. 
Epi-Lipschitz sets in the sense of [R] or [CLS] are epi-Lipschitz in the sense of our definition. Combining Remark 4.6, Example 4.9, Example 4.10, and Theorem 4.12, we obtain

Example 4.13. Any epi-Lipschitz set $B$ has $N L E P(X)$.

In view of Proposition 4.7 (i) and Theorem 4.12, we have

Corollary 4.14. A compact subset $K$ of $X$ is an $\mathcal{L}$-retract provided it satisfies $N L E P(X)$ locally.

We end this section by pointing out that one may also study an "absolute NLE" property. Precisely, we say that a metric space $Y$ has $A N L E P$ if and only if it has $N L E P(Z)$ for every metric space $Z$. We can prove that Theorem 4.12 holds for any metric space (not necessarily compact). The proof involves more detailed considerations. Namely, we show that if an open subset of $Y$ can be represented as the union of pairwise disjoint open sets which have the $N L E P(X)$, then it also has the $N L E P(X)$. This fact together with Lemma 4.11 implies that $N L E P(X)$ is a $G$-hereditary property for $Y$ (see $[\mathrm{M}]$ for the definition of this concept). Our assertion follows from the fact that a local $G$-hereditary property for a metric space is also global (again, see $[M]$ ).

\section{Equilibria ON $\mathcal{L}$-RETRACTS}

We proceed in this last section with the study of equilibria of set-valued maps defined on $\mathcal{L}$-retracts. We start with a lemma which might be of interest on its own.

Lemma 5.1. Let $(X, d)$ be a metric space and $(E,\|\cdot\|)$ a normed space. Let $\Psi$ : $X \longrightarrow 2^{E}$ be a lower semicontinuous map with convex values, and $\Phi: X \longrightarrow 2^{E}$ an upper semicontinuous map with closed convex values such that $\Phi(x) \cap \Psi(x) \neq \emptyset$ for each $x \in X$. Then for any $\delta>0$, there is a continuous map $f: X \longrightarrow E$ such that for every $x \in X$ :

(i) $B(f(x), \delta) \cap \Psi(x) \neq \emptyset$, and

(ii) $\|f(x)-y\|<\delta$ for some $y \in \Phi(\bar{x}), d(x, \bar{x})<\delta$.

Proof. Let us put $U(x)=B_{X}\left(x, \frac{\delta}{2}\right) \cap\left\{x^{\prime} \in X: \Phi\left(x^{\prime}\right) \subset B_{E}\left(\Phi(x), \frac{\delta}{2}\right)\right\}, x \in X$. Let $\mathcal{V}:=\{V\}$ be an open star-refinement of the open cover $\mathcal{U}:=\{U(x)\}_{x \in X}$, i.e. for any $V \in \mathcal{V}$ there is $\bar{x} \in X$ with $s t(V, \mathcal{V}) \subset U(\bar{x})$.

For any $x \in X$, choose $z_{x} \in \Phi(x) \cap \Psi(x)$ and consider the open cover $\mathcal{T}:=$ $\left\{T_{V}(x)\right\}_{V \in \mathcal{V}, x \in V}$ of $X$, where $T_{V}(x):=\left\{x^{\prime} \in V: \Phi\left(x^{\prime}\right) \cap B\left(z_{x}, \frac{\delta}{2}\right) \neq \emptyset\right\}$. Let $\left\{\lambda_{s}\right\}_{s \in S}$ be a locally finite partition of unity subordinated to $\mathcal{T}$. Hence, for each $s \in S$, there are $V_{s} \in \mathcal{V}, x_{s} \in V_{s}$, with $\lambda_{s}\left(x^{\prime}\right)=0$ for $x^{\prime} \notin T_{V_{s}}\left(x_{s}\right)$.

The map $f: X \longrightarrow E$ defined by

$$
f(x):=\sum_{s \in S} \lambda_{s}(x) z_{s}, \quad x \in X,
$$

where $z_{s}=z_{x_{s}}$, is clearly continuous. Moreover, for each $x \in X$ and each index $s$ in the finite set $S(x):=\left\{s \in S: \lambda_{s}(x) \neq 0\right\}$, there exists $z_{s}^{\prime} \in \Psi(x)$ such that $\left\|z_{s}^{\prime}-z_{s}\right\|<\delta$, because $x \in T_{V_{s}}\left(x_{s}\right)$. Thus, by convexity of $\Psi(x)$,

$$
\sum_{s \in S(x)} \lambda_{s}(x) z_{s}^{\prime} \in \Psi(x)
$$


and

$$
\left\|\sum_{s \in S(x)} \lambda_{s}(x) z_{s}^{\prime}-f(x)\right\| \leq \sum_{s \in S(x)} \lambda_{s}(x)\left\|z_{s}^{\prime}-z_{s}\right\|<\delta .
$$

In other words, $B(f(x), \delta) \cap \Psi(x) \neq \emptyset$ for every $x \in X$.

On the other hand, given $x \in X, s \in S(x)$, it follows that $x \in T_{V_{s}}\left(x_{s}\right) \subset V_{s}$, where $x_{s} \in V_{s}$. Since $\mathcal{V}$ is a star-refinement of $\mathcal{U}$, there is $\bar{x} \in X$ such that $x, x_{s} \in U(\bar{x})$. Therefore, $z_{s} \in \Phi\left(x_{s}\right) \subset B(\Phi(\bar{x}), \delta)$ and $\|x-\bar{x}\|<\delta$. The set $B(\Phi(\bar{x}), \delta)$ being convex, we infer that $f(x) \in B(\Phi(\bar{x}), \delta)$.

Remark 5.2. This lemma garantees the existence of a so-called $\delta$-approximate selection for $\Psi$ which is also a $\delta$-approximation of the graph of $\Phi$. As in Michael's selection theorem, assuming that in addition the values of $\Psi$ are closed and $E$ is a Banach space, we conclude that there exists a selection of $\Psi$ which is also a $\delta$-approximation of the graph of $\Phi$.

Given a compact $\mathcal{L}$-retract $K$ in a normed space $(E,\|\|$.$) , let us assume without$ loss of generality that

$$
\begin{gathered}
\exists \beta>0, \exists r: B(K, \beta) \longrightarrow K \text { a retraction, } \exists L>0 \text { such that } \\
\|r(x)-x\| \leq L d(x ; K) \text { for } x \in B(K, \beta) .
\end{gathered}
$$

As in Section 2 (see (A2)), we infer that

$$
\exists \eta>0, \eta<\frac{\beta}{2} \text {, such that }\|r(x)-x\|<\eta \text { for all } x \in B(K, \eta) .
$$

We recalled in the Introduction the definition of the Clarke tangent cone $C_{K}(x)$, $x \in K$. It consists of all those vectors $v \in E$ satisfying the following condition:

$$
\begin{aligned}
\forall\left(x_{n}\right)_{n \in \mathbf{N}} \subset K, x_{n} \rightarrow & x, \forall\left(t_{n}\right)_{n \in \mathbf{N}}, t_{n} \downarrow 0, \exists\left(v_{n}\right)_{n \in \mathbf{N}} \subset E, v_{n} \rightarrow v, \text { such that } \\
& x_{n}+t_{n} v_{n} \in K \text { for all } n \in \mathbf{N} .
\end{aligned}
$$

The set $C_{K}(x)$ is a closed convex cone for each $x \in K$, and $C_{K}(x)=E$ whenever $x \in \operatorname{int}(K)$.

The real function

$$
c(x, v):=\limsup _{\substack{y \rightarrow x, y \in K \\ t \downarrow 0}} \frac{d(y+t v ; K)}{t}
$$

is well-defined $(d(y+t v ; K) / t \leq\|v\|$ for any $y \in K, t>0)$ and satisfies the following properties:

(i) $c: K \times E \longrightarrow \mathbf{R}$ is upper semicontinuous; and

(ii) the function $E \ni v \longmapsto c(x, v)$ is convex for each fixed $x \in K$.

With these properties in mind, we can present the main result of this section.

Theorem 5.3. Let $K$ be a compact $\mathcal{L}$-retract in a normed space $(E,\|\|$.$) with$ $\chi(K) \neq 0$. If $\Phi: K \longrightarrow 2^{E}$ is an upper semicontinuous map with closed convex values satisfying $(2)$, i.e.

$$
\forall x \in K, \Phi(x) \cap C_{K}(x) \neq \emptyset,
$$

then $\Phi$ has an equilibrium. 
Proof. Given $\epsilon>0$ arbitrary, let $\delta:=\frac{\epsilon}{2 L+1}$. Due to the above properties (i)-(ii) of $c$, the map $\Psi: K \longrightarrow 2^{E}$ defined by the formula

$$
\Psi(x):=\{v \in E: c(x, v)<\delta\}, \quad x \in K,
$$

has convex values and its graph is open; it is hence lower semicontinuous. By assumption (2), $\Phi(x) \cap \Psi(x) \neq \emptyset$ for all $x \in K$. In view of Lemma 5.1, there is a continuous $\delta$-approximation $f$ of the graph of $\Phi$ such that

$$
B(f(x), \delta) \cap \Psi(x) \neq \emptyset \text { for all } x \in K .
$$

Since $f$ is continuous it is bounded on $K$, say $\|f(x)\| \leq M$ for some $M>0$. Choose $\tau>0$ with $M \tau<\eta$, where $\eta$ is given by (A5), and a sequence $\left(t_{n}\right)_{n \in \mathbf{N}}$ in $(0, \tau], t_{n} \downarrow 0$. For each $n \in \mathbf{N}$, the map $g_{n}: K \longrightarrow K$ given by

$$
g_{n}(x):=r\left(x+t_{n} f(x)\right), \quad x \in K,
$$

is well-defined since for each $x \in K, d\left(x+t_{n} f(x) ; K\right)<\eta$.

For each $n \in \mathbf{N}$, the homotopy $h_{n}: K \times[0,1] \longrightarrow K$ defined by

$$
h(x, \lambda):=r\left(x+\lambda t_{n} f(x)\right), \quad(x, \lambda) \in K \times[0,1],
$$

joins $g_{n}$ to the identity on $K$. Therefore, $g_{n}$ has a fixed point $x_{n} \in K$. Observe now that

$$
\begin{gathered}
t_{n}\left\|f\left(x_{n}\right)\right\|=\left\|t_{n} f\left(x_{n}\right)\right\|=\left\|x_{n}+t_{n} f\left(x_{n}\right)-g_{n}\left(x_{n}\right)\right\| \\
=\left\|x_{n}+t_{n} f\left(x_{n}\right)-r\left(x_{n}+t_{n} f\left(x_{n}\right)\right)\right\| \leq L d\left(x_{n}+t_{n} f\left(x_{n}\right) ; K\right),
\end{gathered}
$$

where the inequality follows from (A4).

Since $K$ is compact, a subsequence of $\left(x_{n}\right)$ (again denoted by $\left(x_{n}\right)$ ) converges to some $\bar{x} \in K$. In view of $(7)$, there exists $\bar{v} \in \Psi(\bar{x})$ such that $\|\bar{v}-f(\bar{x})\|<\delta$. Hence,

$$
\begin{aligned}
& d\left(x_{n}+t_{n} f\left(x_{n}\right) ; K\right) \leq d\left(x_{n}+t_{n} \bar{v} ; K\right)+t_{n}\left\|\bar{v}-f\left(x_{n}\right)\right\| \\
& \leq d\left(x_{n}+t_{n} \bar{v} ; K\right)+t_{n}\|\bar{v}-f(\bar{x})\|+t_{n}\left\|f(\bar{x})-f\left(x_{n}\right)\right\| .
\end{aligned}
$$

Therefore, for any $n$,

$$
\left\|f\left(x_{n}\right)\right\| \leq L\left(\frac{d\left(x_{n}+t_{n} \bar{v} ; K\right)}{t_{n}}+\|\bar{v}-f(\bar{x})\|+\left\|f(\bar{x})-f\left(x_{n}\right)\right\|\right) .
$$

Letting $n \rightarrow \infty$, we obtain

$$
\begin{gathered}
\|f(\bar{x})\|=\lim _{n \rightarrow \infty}\left\|f\left(x_{n}\right)\right\| \leq L\left(\limsup _{n \rightarrow \infty} \frac{d\left(x_{n}+t_{n} \bar{v} ; K\right)}{t_{n}}+\|\bar{v}-f(\bar{x})\|\right) \\
\leq L(c(\bar{x}, \bar{v})+\|\bar{v}-f(\bar{x})\|)<2 L \delta .
\end{gathered}
$$

Taking into account that $f$ is a $\delta$-approximation of $\Phi$, we infer that there exist $x^{\prime} \in B_{K}(\bar{x}, \delta), y \in \Phi\left(x^{\prime}\right)$ such that $\|y-f(\bar{x})\|<\delta$. Hence, $\|y\|<(2 L+1) \delta=\epsilon$. Since $\epsilon$ is arbitrary and $K$ is compact, it follows that $\Phi$ has a zero.

In view of Examples 4.1, 4.3 and 4.13, we see that Theorem 5.3 generalizes the equilibrium theorems in $[\mathrm{BC}],[\mathrm{P}]$, and $[\mathrm{CLS}]$. Moreover, since every closed convex set is tangentially regular, it follows from Remark 4.2 that Theorem 5.3 also contains Theorem 0 of the Introduction as a particular case.

As before, we obtain the following equilibrium theorem for admissible maps.

Theorem 5.4. Let $K$ be as in Theorem 5.3. If $\Phi: K \longrightarrow 2^{E}$ is an admissible map such that

$$
\Phi(x) \subset C_{K}(x), \text { for all } x \in K,
$$

then $\Phi$ has an equilibrium. 
Proof. As in the preceding proof, there exists $M>0$ such that for each $x \in K$ and each $y \in \Phi(x),\|y\| \leq M$. Choose $\tau>0$ with $M \tau<\eta$, where $\eta$ is given by (A5), and a sequence $\left(t_{n}\right)_{n \in \mathbf{N}}$ in $(0, \tau], t_{n} \downarrow 0$. We define a sequence of maps $\Psi_{n}: K \longrightarrow 2^{K}, n \in \mathbf{N}$, by putting

$$
\Psi_{n}(x):=r\left(x+t_{n} \Phi(x)\right), \quad x \in K .
$$

By Proposition 3.1, each $\Psi_{n}$ is an admissible map. Thus it has a fixed point $x_{n}=r\left(x_{n}+t_{n} y_{n}\right)$, where $y_{n} \in \Phi\left(x_{n}\right)$. Hence,

$$
t_{n}\left\|y_{n}\right\|=\left\|t_{n} y_{n}\right\|=\left\|x_{n}+t_{n} y_{n}-r\left(x_{n}+t_{n} y_{n}\right)\right\| \leq L d\left(x_{n}+t_{n} y_{n} ; K\right) .
$$

Up to a subsequence, $\left(x_{n}, y_{n}\right) \underset{n \rightarrow \infty}{\rightarrow}(\bar{x}, \bar{y})$ in $\operatorname{Graph}(\Phi)$. The upper semicontinuity of $\Phi$ implies that $\bar{y} \in \Phi(\bar{x}) \subset C_{K}(\bar{x})$, i.e. $c(\bar{x}, \bar{y})=0$. We then have

$$
\|\bar{y}\|=\lim _{n \rightarrow \infty}\left\|y_{n}\right\| \leq \limsup _{n \rightarrow \infty} L\left(\frac{d\left(x_{n}+t_{n} y_{n} ; K\right)}{t_{n}}+\left\|\bar{y}-y_{n}\right\|\right)=c(\bar{x}, \bar{y})=0 .
$$

\section{Concluding Remarks}

It is interesting to point out that instead of Lefschetz theory, approximation techniques can be used to prove the main existence results above. For instance, in the proof of Theorem 3.5, one can observe that the map $\Psi_{n}$ is the composition of the continuous retraction $r$ after a convex valued upper semicontinuous map. Such maps are approximable in the sense of the graph (see [K2] and [B]) and admit fixed points on suitable compact domains (Example 3.4 can be modified as to hold for such maps).

It is also natural to ask whether Theorems 5.3, 5.4 hold true with the larger Bouligand cone. Obviously, if the set $K$ in those theorems is tangentially regular, both cones coincide. However, as the next example shows, for non-regular sets, Theorem 5.3 is in general false with Bouligand's cone.

Example 6.1. Consider the restriction of the function $f$ of Example 1.1 of the Introduction to the subset $K_{+}$of $\mathbf{R}^{2}$ consisting of the upper half of the set $K$ considered in that example. Clearly, $f$ has no zero and $K_{+}$is bi-Lipschitz homeomorphic to the compact convex set $[-2,2] \times\{0\}$; hence $K_{+}$is an $L$-retract. However, $C_{K_{+}}(0,0)=\{0\} \not \supset f(0,0)=(0,1) \in T_{K_{+}}(0,0)$.

It is important to mention that the existence of equilibria on neighborhood retracts depends strongly on the choice of the retraction, as the following result suggests.

Proposition 6.2. Let $(E,\langle.,\rangle$.$) be a Hilbert space and K \subset E$ be a compact neighborhood retract with nontrivial Euler characteristic and with a given retraction $r: U \longrightarrow K$ ( $U$ being a neighborhood of $K$ ). If $\Phi: K \longrightarrow 2^{K}$ is an upper hemicontinuous map with closed convex values such that

$$
\forall x \in K, \forall p \in r^{-1}(x), \inf _{y \in \Phi(x)}\langle p-x, y\rangle \leq 0,
$$

then $\Phi$ has an equilibrium in $K$.

Proof. Exactly as in the proof of Theorem 3.5, we construct a continuous map $f: K \longrightarrow E$ such that

$$
\sup _{y \in \Phi(x)}\langle-f(x), y\rangle<0 \text { for all } x \in K .
$$


Let $\mu>0$ be as in (A1) and satisfying $\|\mu f(x)\|<\delta$. The map $g: K \longrightarrow K$ given by

$$
g(x):=r(x+\mu f(x)) \text { for } x \in K
$$

is well-defined and homotopic to the identity on $K$ (through the homotopy $h(x, \lambda)$ $:=r(x+\lambda \mu f(x)),(x, \lambda) \in K \times[0,1])$. Thus there exists $\bar{x} \in K$ such that $\bar{x}=$ $g(\bar{x})=r(\bar{x}+\mu f(\bar{x}))$, i.e. $\bar{x}+\mu f(\bar{x}) \in r^{-1}(\bar{x})$. Hence, $\inf _{y \in \Phi(\bar{x})}\langle f(\bar{x}), y\rangle \leq 0$. This contradiction ends the proof.

We end this paper with an open problem. Let us recall that for nonconvex maps defined on $L$-retracts with non-trivial Euler characteristic, we were able to formulate an existence result with the strong tangency condition $\Phi(x) \subset C_{K}(x)$ (Theorem 5.4). It is natural to pose:

Conjecture 6.3. Assume that $K$ is as in Theorem 5.3 and that it is tangentially regular. If $\Phi: K \longrightarrow 2^{E}$ is an upper semicontinuous map with acyclic values (an admissible map) satisfying

$$
\Phi(x) \cap C_{K}(x) \neq \emptyset, \text { for all } x \in K,
$$

then $\Phi$ has an equilibrium.

We believe that different techniques (we have in mind homotopy approximation techniques developed in [K2]) than the one used in this paper are needed in order to provide a positive answer to this conjecture for some classes of acyclic maps.

\section{REFERENCES}

[A] Arens R., Extensions of functions on fully normal spaces, Pacific J. Math. 6 (1952), 11-22. MR 14:191h

[AC] Aubin J. P. and A. Cellina, Differential inclusions, Springer-Verlag, Berlin, 1984. MR 85j:49010

[AF] Aubin J. P. and H. Frankowska, Set-valued analysis, Birkhäuser, Boston, 1990. MR 91d:49001

[B] Ben-El-Mechaiekh H., Continuous approximation of multifunctions, fixed points and coincidences, Proceedings of the Second International Conference on Approximation and Optimization in the Carribean, M. Florenzano et al. Eds., Approximation and Optimization Vol. 8, Peter Lang, Frankfurt, 1995, pp. 69-97. MR 96k:47091

[BC] Bonnisseau J-M. and B. Cornet, Fixed-point theorems and Morse's lemma for lipschitzian functions, J. Math. Anal. Appl. 146 (1990), 318-332. MR 91c:58014

[BK] Ben-El-Mechaiekh H. and W. Kryszewski, Equilibrium for perturbations of upper hemicontinuous set-valued maps by convex processes, Georgian Math. J. 3 (1996), 201-205. MR 97c:34025

[Bo] Borsuk K., Theory of retracts, Monografle Matematyczne 44, Warszawa, 1967. MR 35:7306

[Br] Browder F., The fixed point theory of multivalued mappings in topological vector spaces, Math. Ann. 177 (1968), 283-301. MR 37:4679

[C] Cornet B., Paris avec handicaps et théorèmes de surjectivité de correspondances, C. R. Acad. Sc. Paris Sér. A 281 (1975), 479-482. MR 52:7577

[CLS] Clarke F. H., Y. S. Ledyaev and R. J. Stern, Fixed points and equilibria in nonconvex sets, Nonlinear Anal. 25 (1995), 145-161. MR 96g:49005

[D] Dugundji J., An extension of Tietze's theorem, Pacific J. Math. 1 (1951), 353-367. MR 13:373c

[F1] Fan K., Fixed point and minimax theorems in locally convex topological spaces, Proc. Nat. Acad. Sc. USA 38 (1952), 121-126. MR 13:858d

[F2] Fan K., Some properties of convex sets related to fixed point theorems, Math. Ann. 266 (1984), 519-537. MR 85i:47060

[G] Górniewicz L., Homological methods in fixed-point theory of multivalued maps, Diss. Math. CXXIX, Warsawa, 1976. MR 52:15438 
[Gr] Granas A., Points fixes pour les applications compactes: espaces de Lefschetz et la théorie de l'indice, SMS, Presses de l'Université de Montréal, Montréal, 1980. MR 81i:55002

[H] Hu S-T., Theory of Retracts, Wayne State Univ. Press, Detroit, 1965. MR 31:6202

[K1] Kryszewski W., Topological and approximation methods in degree theory of set-valued maps, Diss. Math. 336 (1994). MR 95m:55005

[K2] Kryszewski W., Some homotopy classification and extension theorems for the class of compositions of acyclic set-valued maps, Bull. Sci. Math. 119 (1995), 21-48. MR 95m:55012

[L] Lassonde M., On the use of KKM multifunctions in fixed point theory and related topics, J. Math. Anal. Appl. 97 (1983), 151-201. MR 84k:47049

[LR] Lasry J. M. and R. Robert, Analyse nonlinéaire multivoque, Cahiers de Math. de la Décision 7611, Université de Paris-Dauphine, Paris, 1976.

[M] Michael E., Local properties of topological spaces, Duke Math. J. 21 (1954), 163-171. MR 15:977c

[P] Plaskacz S., On the solution sets of differential inclusions, Boll. U. M. I. (7) 6-A (1992), 387-394. MR 93m:34021

[R] Rockafellar R. T., Clarke's tangent cones and boundaries of closed sets in $\mathbf{R}^{n}$, Nonlinear Analysis 3 (1979), 145-154. MR 80d:49032

[S] Spanier E., Algebraic topology, McGraw-Hill, New York, 1966. MR 35:1007

Department of Mathematics, Brock University, St. Catharines, Ontario, L2S 3A1, CANADA

Current address: Department of Mathematics, Sultan Qaboos University, P.O. Box 50, AlKhod, Oman

Instytut Matematyki, Uniwersitet Mikolaja Kopernika, ul. Chopina 12/18, 87-100 TORuń, POLAND

E-mail address: wkrysz@mat.uni.torun.pl and wkrysz@plunlo51.bitnet 